\title{
BAIRE SETS, BOREL SETS AND SOME TYPICAL SEMI-CONTINUOUS FUNCTIONS
}

\author{
KEIÔ NAGAMI
}

Recently Hing Tong [3], M. Katětov [4] and C. H. Dowker [2] have established two sorts of insertion theorems for semi-continuous functions defined on normal and countably paracompact normal spaces. The purpose of this paper is to give the insertion theorem for some typical semi-continuous functions defined on a $T$-space. The relations between Baire sets and Borel sets in some topological spaces are also studied.

Throughout this paper, unless in a special context we explicitly say otherwise, any function defined on a space is real-valued and a sequence of functions means a countable sequence of them. A family of functions defined on a space $R$ is called complete if the limit of any sequence of functions in it is also contained in it. The minimal complete family of functions defined on $R$ which contains all continuous functions is called the family of Baire functions and its element a Baire function. A subset of $R$ is called a Baire set if its characteristic function is a Baire function. A subset of $R$ is called respectively elementaryopen or elementary-closed if it is written as $\{x ; f(x)>0\}$ or $\{x ; f(x) \geqslant 0\}$ by a suitable continuous function $f(x)$ defined on $R$. The minimal completely additive class of sets which contains all open sets in $R$ is called the family of Borel sets and its element a Borel set. It is well known that the family of Borel sets contains the family of Baire sets and that the family of Baire sets coincides with the minimal completely additive class of sets which contains all the elementary open (closed) sets.

THEOREM 1. In oder that a subset $A$ of a $T$-space $R$ is elementary-open or elementary-closed, it is necessary and sufficient that the characteristic function $\varphi_{A}(x)$ of $A$ is the limit of a monotonically increasing or decreasing sequence of continuous functions, respectively.

Received February 5, 1954. 
Proof. Let $A$ be an elementary closed set:

$$
A=\{x ; f(x) \geq \lambda\},
$$

where $f(x)$ is a continuous function defined on $R$. As the real line is homeomorphic to the open interval $(1,2)$ preserving the order, we can assume with no loss of generality that $1<f(x)<2$ everywhere and $\lambda>0$. Let $h(x)=\min$ $(f(x), \lambda) / \lambda$ and $h(x), h^{2}(x), \ldots \downarrow \varphi_{A}(x)$ where $h^{i}(x)$ is evidently continuous. Thus $\varphi_{A}(x)$ is the limit of a monotonically decreasing sequence of continuous functions. When $A$ is elementary-open, $R-A$ is elementary-closed. Let $f_{\imath}(x)$ be continuous functions such that $f_{i}(x) \downarrow \varphi_{R-A}(x)$. And then $1-f_{i}(x) \uparrow 1-\varphi_{R-A}(x)$ $=\varphi_{A}(x)$. Thus $\varphi_{A}(x)$ is the limit of a monotonically increasing sequence of continuous functions.

Conversely let $A$ be the set, the characteristic function $\varphi_{A}(x)$ of which is the limit of a monotonically increasing sequence of continuous functions $f_{i}(x)$ defined on $R$, where we can assume with no loss of generality that $0 \leqq f_{i}(x) \leqq 1$ everywhere. Let

$$
f(x)=\sum_{i=1}^{\infty} f_{i}(x) / 2^{i}
$$

Then $f(x)$ is evidently a continuous function. It is not hard to verify that $A=\{x ; f(x)>0\}$. Thus $A$ is elementary-open. When $A$ is a set, the characteristic function $\varphi_{A}(x)$ of which is the limit of a monotonically decreasing sequence of continuous functions, $R-A$ is elementary-open by the above argument and $A$ is an elementary closed set. Q.E.D.

This theorem shows that elementary-open and elementary closed sets are Baire sets.

Corollary. A countable sum and a finite intersection of elementary open sets are elementary-open. A countable intersection and a finite sum of elementary closed sets are elementary-closed.

This can be easily seen from the above theorem.

THEOREM 2. If $f(x)$ defined on a T-space $R$ is the limit of a monotonically increasing sequence of continuous functions $f_{i}(x),\{x ; f(x)>\lambda\}$ becomes an elementary open set for any $\lambda$. If $f(x)$ is the limit of a monotonically decreasing sequence of continuous functions, $\{x ; f(x) \geq \lambda\}$ becomes an elementary closed 
set for any $\lambda$.

Proof. We shall only prove the first half, since the last half of the theorem is proved by the dual argument to the first one. Since $\{x ; f(x)>\lambda\}=\{x ; f(x)$ $-\lambda>0\}$ and $f_{i}(n)-\lambda \uparrow f(x)-\lambda$, it is sufficient only to prove that $\{x ; f(x)>0\}$ is elementary-open. Let $A_{i}=\left\{x ; f_{i}(x)>0\right\}$. We have $A=\bigcup_{i=1}^{\infty} A_{i}$. Since $A_{i}$ are all elementary-open, $A$ is also elementary-open by the corollary of Theorem 1. Q.E.D.

The converse of this theorem also holds as follows.

THEOREM 3. Let $f(x)$ be a function defined on a T-space $R$. If $\{x ; f(x)$ $>\lambda\}$ is elementary-open for any $\lambda, f(x)$ becomes the limit of a monotonically increasing sequence of continuous functions. If $\{x ; f(x) \geqslant \lambda\}$ is elementaryclosed for any $\lambda, f(x)$ becomes the limit of a monotonically decreasing sequence of continuous functons.

Proof. We shall only prove the first half, since the last half can be proved by the dual argument to the first. Let $\varphi(t)$ be the order-preserving homeomorphism of the real line onto the open interval $(0,1)$, and then

$$
\{x ; f(x)>\lambda\}=\{x ; \varphi(f(x))>\varphi(\lambda)\} .
$$

If $\varphi(f(x))$ is the limit of a monotonically increasing sequence of continuous functions, $f(x)$ has the same property. Hence it suffices to consider the case where $0<f(x)<1$. Let $g_{j}(x)$ be the characteristic function of $\{x ; f(x)>j / n\}$ where $n$ is an arbitrary positive integer. And then, by Theorem $1, g_{j}(x)$ is the limit of a monotonically increasing sequence of continuous functions. Let

$$
f_{n}(x)=\left(\sum_{j=1}^{n-1} g_{j}(x)\right) / n,
$$

then we can easily see that $f_{n}(x)$ is the limit of a monotonically increasing sequence of continuous functions and that $\left|f_{n}(x)-f(x)\right| \leqq 1 / n$ everywhere. Thus $f(x)$ is the limit of uniformly convergent sequence $\left\{f_{n}(x)\right\}$ and so is the limit of a monotonically increasing sequence of continuous functions. Q.E.D.

THEOREM 4. If $A$ and $B$ are disjoint elementary closed sets of a $T$-space, there are elementary open sets $U$ and $V$ such that $A \subset U, B \subset V$ and $U \cap V=\phi$ (the empty set). Moreover, there is a continuous function $f(x)$ defined on the space such that $A \subset\{x ; f(x)=0\}, B \subset\{x ; f(x)=1\}$. 
Proof. Let $A=\left\{x ; f_{1}(x) \geqslant 0\right\}$ and $B=\left\{x ; f_{2}(x) \geqslant 0\right\}$, where $f_{1}(x)$ and $f_{2}(x)$ are continuous functions defined on the space. Then $U=\left\{x ; f_{1}(x)-f_{2}(x)\right.$ $>0\}$ and $V=\left\{x ; f_{1}(x)-f_{2}(x)<0\right\}$ are the desired sets. The last half of the theorem can be proved analogously to the proof of the Urysohn lemma. Q.E.D.

As an immediate consequence of Theorems 2, 3 and 4, we get the following result established by Hing Tong [3].

COROLlaRY 1 (Hing Tong). In a $T$-space $R$ the following conditions are equivalent :

i) $R$ satisfies the $T_{4}$-separation axiom and every closed set in $R$ is a $G_{\delta}$-set.

ii) Every closed set in $R$ is an elementary closed set.

iii) Every lower semi-continuous function defined on $R$ is the limit of a monotonically increasing sequence of continuous functions.

COROLlARY 2. If a $T$-space $R$ satisfies any one of the conditions stated in the preceding corollary, the family of Borel sets coincides with the family of Baire sets.

This can easily verified from ii) of the preceding corollary and from the well-known fact that the family of Baire sets coincides with the minimal completely additive class of sets which contains all the elementary open (closed) sets.

THEOREM 5. If a space $R$ is regular and has the Lindelöf property, an open set $G$ in $R$ is elementary-open if and only if $G$ has the Lindelof property.

Proof. If $G$ is elementary-open, $G$ is an $F_{\sigma}$-set. Since any closed subset of $R$ has obviously the Lindelöf property, $G$ has also the same property as a countable sum of sets with the property.

Conversely, if $G$ is an open set with the Lindelöf property, $G$ has the starfinite property ([4, Theorem 10]). Hence $G$ is a paracompact $T_{2}$-space and is, therefore, normal ([1, Theorem 1]). We can construct the covering $\left\{U_{\alpha}\right\}$ of $G$, the elements of which are all elementary-open. On account of the Lindelof property of $G$, there is a countable sub-family $\left\{U_{i}\right\}$ of $\left\{U_{\alpha}\right\}$ such that $G=\bigcup_{i=1}^{\infty} U_{i}$. Thus $G$ becomes an elementary open set as a countable sum of elementary open sets. Q.E.D.

COROLLARY 1. If $R$ satisfies the same conditions in the theorem, the family of Baire sets is contained in the completely additive class of sets generated by 
an arbitrary open basis of $R$.

This is obvious from the theorem.

Corollary 2. Let $R$ be a paracompact $T_{2}$-space with the local Lindelöf property $^{1 j}$ and let $\left\{U_{\alpha}\right\}$ be an arbitrary open basis of $R$. Then every Baire set is a sum of sets which are elements of the completely additive class of sets generated by $\left\{U_{\alpha}\right\}$ and together form a discrete collection. ${ }^{2}$

Proof. Let $A$ be an arbitrary Baire set in $R$ and let $U$ be the completely additive class of sets generated by $\left\{U_{\alpha}\right\} . \quad R$ can be decomposed into mutually disjoint open sets $\left\{R_{3}\right\}$ each of which has the Lindelöf property by [1, Theorem 7] and [5, Theorem 11]. Since the characteristic function of each $R_{\beta}$ is continuous, $R_{\beta}$ is a Baire set. Hence $A \cap R_{\beta}=A_{\beta}$ is a Baire set and $A_{\beta} \in U$ by the above corollary. It can be easily seen that $\left\{A_{\beta}\right\}$ forms a discrete collection. Q.E.D.

THEOREM 6. Every countably compact Baire set in a T-space $R$ is an elementary closed set.

In order to prove this we begin with the following

Lemma. ${ }^{3)} \quad$ For every Baire function $f(x)$ defined on a $T$-space $R$, there exists a sequence of continuous functions $f_{i}(x)$ defined on $R$ such that $f(x)=f(y)$ whenever $f_{i}(x)=f_{i}(y)$ for all $i$. In other words every Baire function is determined by a countable number of continuous functions.

Proof. Let $\mathfrak{F}$ be the family of all the functions which are determined by a countable number of continuous functions. Let $f(x)$ be the limit of a sequence of functions $f_{i}(x) \in \tilde{j}$. If $\left\{f_{i j}(x) ; j=1,2, \ldots\right\}$ is a sequence of continuous functions which determines $f_{i}(x),\left\{f_{i j}(x) ; i, j=1,2, \ldots\right\}$ determines $f(x)$. Hence $f(x) \in \tilde{F}$, which shows the completeness of $\mathfrak{F}$. Since $\tilde{f}$ contains all the continuous functions defined on $R, \tilde{F}$ contains all the Baire functions defined on $R$. Q.E.D.

Proof of Theorem. Let $F$ be a Baire set which is countably compact

1) A space is called to have the local Lindelöf property, if each point has a neighbourhood with the Lindelof property.

2) A collection of sets is called discrete, if the closures of these sets are mutually disjoint and any sub-collection of these closures has a closed sum.

3. This lemma was stated in the lecture of Prof. K. Itô at Nagoya University. 
and let $\left\{f_{i}(x)\right\}$ be a sequence of continuous functions defined on $R$ which determines $\varphi_{F}(x)$, the characteristic function of $F$, in the sense of the lemma. Let

$$
\rho(x, y)=\sum_{i=1}^{\infty}\left|f_{i}(x)-f_{i}(y)\right| / 2^{i}\left(1+\left|f_{i}(x)-f_{i}(y)\right|\right) .
$$

And then $\rho$ gives a general metric on $R$. By means of $\rho, R$ is decomposable into classes $x^{*}, y^{*}, \ldots$, where

$$
x^{*}=\left\{x^{\prime} ; \rho\left(x, x^{\prime}\right)=0\right\} .
$$

We consider $R^{*}=\left\{x^{*}\right\}$ as a metric space with the metric $\rho^{*}$ :

$$
\rho^{*}\left(x^{*}, y^{*}\right)=\rho(x, y), \quad \text { where } x \in x^{*}, y \in y^{*} .
$$

$\rho^{*}$ is clearly independent of the choice of $x$ and $y$. The mapping $g(x)=x^{*}$ from $R$ onto $R^{*}$ is continuous, since $\rho(x, y)$ is a continuous function defined on $R$ as a function of $y$ for every fixed $x \in R . \quad F^{*}=\{g(x) ; x \in F\}$ is the continuous image of coutably compact $F$ and is also countably compact. Hence $F^{*}$ is closed in $R^{*}$. By Corollary 1 of Theorem $4, F^{*}$ is an elementary closed set and is expressible as

$$
F^{*}=\left\{x^{*} ; f^{*}\left(x^{*}\right) \gtrsim 0\right\},
$$

where $f^{*}\left(x^{*}\right)$ is continuous on $R^{*}$. Let $f(x)=f^{*}(g(x))$, then $f(x)$ becomes continuous on $R . \quad \rho(x, y)=0$ implies $f_{i}(x)=f_{i}(y)$ for all $i$ and hence $\varphi_{F}(x)$ $=\varphi_{F}(y)$. In other words $g^{-1}\left(x^{*}\right) \cap F \neq \phi$ implies $g^{*}\left(x^{*}\right) \subset F$. Therefore,

$$
F=\{x ; f(x) \geqslant 0\}
$$

and is an elementary closed set. Q.E.D.

Corollary. A locally countably compact $T_{1}$-group $G$ is metrizable if and only if the family of Borel sets coincides with the family of Baire sets.

Proof. 'Only if' part has been already shown in Corollary 2 of Theorem 4.

'If' part: Let $e$ be the unit element of $G$. Since $e$ is obviously countably compact, $e$ is a $G_{\delta}$-set by the assumption and the above theorem. Now $e$ is represented as

$$
e=\bigcap_{i=1}^{\infty} V_{i}
$$

where $\vec{V}_{1}$ is countably compact and every $V_{i}$ is open. Then it can be easily 
seen that $\left\{V_{i}\right\}$ forms a complete system of neighbourhoods of $e$ from the countable compactness of $\bar{V}_{1}$. Hence $G$ is metrizable. Q.E.D.

THEOREM 7. Let $\left\{U_{i}\right\}$ be a countable covering of a $T$-space $R$, the elements of which are all elementary-open. Then there is a locally finite refinement $\left\{V_{i}\right\}$, the elements of which are all elementary-open so that $V_{i} \subset U_{i}$ for every $i$.

Proof. Since $\bigcup_{k=1}^{i} U_{k}$ is elementary-open, $\bigcup_{k=1}^{i} U_{k}=\left\{x ; f_{i}(x)>0\right\}$, where $f_{i}(x)$ is a continuous function defined on $R$. Let

$$
\begin{aligned}
& U_{i j}=\left\{x ; f_{i}(x)>1 / j\right\}, \\
& U_{i j}^{\prime}=\left\{x ; f_{i}(x) \geq 1 / j\right\},
\end{aligned}
$$

then it can easily be seen that

$$
\begin{aligned}
& \bigcup_{k=1}^{i} U_{k}=\bigcup_{j=1}^{\infty} U_{i j}=\bigcup_{j=1}^{\infty} U_{i j}^{\prime}, \\
& U_{i j} \subset U_{i j}^{\prime} \subset U_{i, j+1} .
\end{aligned}
$$

Let

$$
V_{i}=U_{i}-\bigcup_{j=1}^{i-1} U_{j i}^{\prime}
$$

then $V_{i}$ is elementary-open. $\left\{V_{i}\right\}$ is nothing but we seek for. Let $x$ be an arbitrary point of $R$ and let $U_{i}$ be the first element of $\left\{U_{i}\right\}$ which contains $x$. Then

$$
x \in U_{i}-\bigcup_{j=1}^{i-1} U_{j} \subset U_{i}-\bigcup_{j=1}^{i-1} U_{j i}^{\prime}
$$

and $\left\{V_{i}\right\}$ is a covering of $R$. If $x \in U_{i}$, there is a $j$ such that $x \in U_{i j}$. If $k>i, j$,

$$
V_{k} \cap U_{i j} \subset\left(U_{k}-\bigcup_{l=1}^{k-1} U_{l k}^{\prime}\right) \cap U_{i j}^{\prime}=\phi
$$

This shows that $U_{i j}$ meets only a finite number of sets $V_{k}$. Hence $\left\{V_{i}\right\}$ is locally finite. Since clearly $V_{i} \subset U_{i}$ for every $i,\left\{V_{i}\right\}$ satisfies the conditions stated in the theorem. Q.E.D.

THEOREM 8. Let $g(x)$ and $h(x)$ be respectively the limit of a monotonically decreasing sequence and a monotonically increasing sequence of continuous functions defined on a T-space $R$ such that $g(x)<h(x)$ everywhere. Then there is a continuous function $f(x)$ defined on $R$ such that $g(x)<f(x)<h(x)$ everywhere. 
Proof. (Our proof is an application of Dowker's method [2, Theorem 4].) The rational numbers are countable. Denote them by $\left\{r_{i}\right\}$. Let $U_{i}=\{x ; g(x)$ $\left.<r_{i}<h(x)\right\}$. Then

$$
U_{i}=\left(R-\left\{x ; g(x) \geqq r_{i}\right\}\right) \cap\left\{x ; h(x)>r_{i}\right\} .
$$

Hence $U_{i}$ is elementary-open by Theorem 2. Since $g(x)<h(x)$ for every $x \in R$, there is a rational number $r_{i(x)}$ with $g(x)<r_{i(x)}<h(x)$. Hence $x \in U_{i(x)}$. Thus $\left\{U_{i}\right\}$ is a covering of $R$, the elements of which are all elementary-open. By Theorem 7 there is a locally finite covering $\left\{V_{i}\right\}$ of $R$, the elements of which are all elementary-open so that $V_{i} \subset U_{i}$ for every $i$. Since $\left\{V_{i}\right\}$ is a covering of $R, V_{1}$ contains an elementary closed set $W_{1}=R-\bigcup_{i=2}^{\infty} V_{i}$. By Theorem 4 , there are an elementary closed set $A_{1}$ and an elementary open set $B_{1}$ such that $V_{1} \supset A_{1} \supset B_{1} \supset W_{1}$. Then

$$
\left\{B_{1}, V_{i} ; i \geq 2\right\}
$$

is a covering of $R$, the elements of which are all elementary-open. Repeating this procedure, we can construct, for any $j$, a covering

$$
\left\{B_{1}, B_{2}, \ldots, B_{j}, V_{j+2}, V_{j+2}, \ldots\right\}
$$

of $R$, the elements of which are all elementary-open so that $V_{k} \supset B_{k}$ and $V_{k} \supset A_{k} \supset B_{k}$ for some elementary closed $A_{k}$ and for any $k \leqq j$. Then $\left\{B_{i} ; i=1\right.$, $2, \ldots\}$ is a covering of $R$; for every point $x \in R$ there is the maximal number $i(x)$ such that $x \in V_{i(x)}$, since $\left\{V_{i}\right\}$ is a locally finite covering of $R$. $x \notin \bigcup_{j=1}^{i(x)} B_{j}$ leads to a contradictory fact that $\left\{B_{1}, \ldots, B_{i(x)}, V_{i(x)+1}, \ldots\right\}$ is not a covering of $R$. Hence $\left\{B_{i}\right\}$ is a covering of $R$ which has evidently the locally finite property. There are, by Theorem 4, continuous functions $f_{i}(x)$ defined on $R$ such that $f_{i}(x)=r_{i}$ for $x \in A_{i}, f_{i}(x)=-\infty$ for $x \in R-V_{i}$ and $-\infty \leqq f_{i}(x) \leqq r_{i}$ for $x \in R$. Putting

$$
f(x)=\sup _{i} f_{i}(x)
$$

we get a function defined on $R$. Each point $x_{0} \in R$ is contained in some open set $G\left(x_{0}\right)$ which meets only a finite number of sets $V_{i}$. Hence, in $G\left(x_{0}\right)$, for all but a finite number of values of $i, f_{i}(x)=-\infty$. Thus, in each $G\left(x_{0}\right)$, $f(x)$ is the supremum of a finite number of continuous functions. Hence $f(x)$ is a continuous function defined on $R$. In $U_{i}, f_{i}(x) \leqq r_{i}<h(x)$ and, in $R-U_{i}$, 
$f_{i}(x)=-\infty<h(x)$. Thus $f_{\imath}(x)<h(x)$ everywhere and, for every $x \in R, f(x)$ is the supremum of a finite number of $f_{i}(x)$, each of which is less than $h(x)$. Therefore $f(x)<h(x)$ everywhere. Each $x$ is in some $B_{i}$ and, for this $i, f_{i}(x)$ $=r_{i}$; hence $g(x)<r_{i}=f_{i}(x) \leqq f(x)$ and $g(x)<f(x)$ everywhere. Therefore $g(x)<f(x)<h(x)$ everywhere. Q.E.D.

If we replace ' $\leqq$ ' instead of ' $<$ ' in the above, the theorem thus obtained holds also: This is a trivial consequence of Hing Tong's result [3, Theorem 1].

\section{BIBLIOGRAPHY}

[1] J. Dieudonné : Une généralisation des espaces compacts, Journ. Math. Pures et Appl., 23 (1944), 65-76.

[2] C. H. Dowker: On countably paracompact spaces, Canadian J. Math., 3 (1951), 219-224.

[3] Hing Tong: Some characterizations of normal and perfectly normal spaces, Duke Math. J., 19 (1952), 289-292.

[4] M. Katětov: On real-valued functions in topological spaces, Fund. Math., 38 (1951), 85-91.

[5] K. Morita: Star-finite coverings and the star-finite property, Mathematica Japonicae 1 (1948), 60-68. 
